\title{
Effect of the anisotropic permeability in the frequency dependent properties of the superficial layer of articular cartilage.
}

\author{
Dario Gastaldi ${ }^{1}$, Matteo Taffetani ${ }^{2}$, Roberto Raiteri ${ }^{3,4}$ and Pasquale Vena ${ }^{1}$ \\ ${ }^{1}$ Department of Chemistry, Materials and Chemical Engineering, Politecnico di Milano, Italy \\ ${ }^{2}$ Mathematical Institute, University of Oxford, UK \\ ${ }^{3}$ Department of Informatics, Bioengineering, Robotics and System Engineering, University of Genova, Italy \\ ${ }^{4}$ Istituto di Biofisica, Consiglio Nazionale delle Ricerche, Genova, Italy
}

October 25, 2018

\begin{abstract}
Articular cartilage is a tissue of fundamental importance for the mechanics of joints, since it provides a smooth and lubricated surface for the proper transfer of loads. From a mechanical point of view, this tissue is an anisotropic poroviscoelastic material: its characteristics at the macroscopic level depend on the complex microscopic architecture. With the ability to probe the local microscopic features, dynamic nanoindentation test is a powerful tool to investigate cartilage mechanics. In this work we focus on a length scale where the time dependent behaviour is regulated by poroelasticity more than viscoelasticity and we aim to understand the effect of the anisotropic permeability on the mechanics of the superficial layer of the articular cartilage. In a previous work, a finite element model for the dynamic nanoindentation test has been presented. In this work, we improve the model by considering the presence of an anisotropic permeability tensor that depends on the collagen fibers distribution. Our sensitivity analysis highlights that the permeability decreases with increasing indentation, thus making the tissue stiffer than the case of isotropic permeability, when solicited at the same frequency. With this improved model, a revised identification of the mechanical and physical parameters for articular cartilage is provided. To this purpose the model was used to simulate experimental data from tests performed on bovine tissue, giving a better estimation of the anisotropy in the elastic properties. A relation between the identified macroscopic anisotropic permeability properties and the microscopic rearrangement of the fiber/matrix structure during indentation is also provided.
\end{abstract}

Keywords: Articular cartilage; Dynamic Nanoindentation; Anisotropic permeability; Poroelasticity; Finite Element.

\section{Introduction}

The study of the mechanical and physical properties of Articular Cartilage (AC) is a field of great interest in both clinical applications and bio-engineering. Indeed, the through-the-thickness inhomogeneity and the anisotropic orientation of its fibrillar components at the microscale give to the tissue a non-linear time- and depth-dependent response at the macroscale, which is essential for the correct functionality of the joints in terms of load transfer and lubrication support [13]. 
Since 65 to $85 \mathrm{w} / \mathrm{w} \%$ of cartilage is water, the fluid phase plays a relevant physiologic role in determining the tissue compressive properties, both providing the mechanical stimulus for the cells growth and regulating the nutrient transport [11]. The macroscopic permeability of AC is affected by the micro-architecture of the cartilage. As the cartilage is loaded, water is forced to flow through the tissue in a way that results from the combined effect of (i) the solid matrix porosity and (ii) the glycosaminoglycan molecules (GAGs) attraction that hinders the outward flow of water molecules $[12,14]$. The fiber network affects the capability of the fluid to flow according to the pressure gradient, providing preferential directions: indeed, fluid flows more easily along the direction parallel to fibers than perpendicularly to them. Since the architecture of the fibers changes substantially across the thickness, also the permeability is expected to change accordingly: a higher permeability in the direction parallel to the articular surface is expected in the superficial layers; a roughly isotropic permeability characterizes the middle zone; in the deep zone, where the fibers are perpendicular to the tide mark, the permeability along the axial (through-the-thickness) direction is higher than that along the transverse one [3].

The mutual interaction of the dense network of GAGs gives rise to an osmotic pressure that leaves empty spaces filled with fluid that flows through it [14]. Hence, the hydraulic permeability is expected to be isotropic if the geometrical arrangement of GAGs is random.

When AC is loaded, its microstructure changes and, as a consequence, its intrinsic permeability is affected. For example, compression tests on cylindrical samples of bovine cartilage have shown that an increase in the compression leads to an overall decrease in the permeability, more dramatically in the radial than in the axial direction: for strain compression of 20-40\% Reynaud and Quinn [15] measured a hydraulic permeability tensor with a strong anisotropy (a 10-fold difference between axial and radial directions).

In the present study, the permeability of the tissue is studied by means of dynamic nanoindention tests $[7,5,21]$ using an Atomic Force Microscope (AFM) with a spherical tip at the end of a flexible cantilever. When a given harmonic oscillation is applied to the free end of the cantilever, the spherical tip at the other end of the cantilever penetrates into the tissue with harmonic time variation (input). The time history of the reaction force at the tip of the cantilever is recorded as the output signal and the frequency dependent properties of the tissues are obtained by post-processing the amplitude of the sinusoidal reaction force and the phase shift between the input and output.

The AFM-based nanoindentation technique has already proven to be effective in determining the time- or frequency-dependent properties of soft hydrated tissues: different characteristic lengths can be tested by changing the tip radius or the penetration depth $[9,8,17]$. Furthermore, also anisotropic elastic properties of AC could be assessed through this relatively simple mechanical test $[21]$.

As the tissue may exhibit both flow-independent viscoelasticity and poroelasticity responses depending on the loading conditions [10], Han et al. [6] have shown that cartilage tissue is viscoelastic at small scales (when the collagen fiber is probed with the tip) and poroelastic at larger characteristic lengths. In the present work, loading conditions entailing mostly poroelastic response of the tissue will be considered.

This introductory discussion highlighted the fundamental role of the permeability in the mechanics of the articular cartilage and the importance of a proper estimation of its anisotropic characteristics. The aim of this work is to provide a quantitative description of the anisotropic permeability in the superficial layers of bovine articular cartilage when tested using dynamic nanoindentation. The model presented in Taffetani et al. [18] is here enriched by a description of the anisotropic permeability tensor, derived from local microscopic considerations. We first perform a sensitivity analysis to evaluate the effect of a transversely anisotropic permeability tensor on the response of the cartilage under a wide range of controlled conditions, i.e. when radii and depths are varied. Then we consider three sets of experimental data described elsewhere [21] and we perform a pa- 
rameters identification by fitting the experimental data with the outcomes of this enriched finite element model.

\section{Materials and Methods}

In the typical dynamic indentation test that we want to simulate here, a spherical tip of radius $R$ is put in contact with a cylindrical sample of radius $B$ and height $H$ and a preload is applied by imposing an initial given penetration depth. Then, the tip is forced to oscillate harmonically at a given frequency $f$ and a given amplitude: once a stationary response is achieved, the force oscillation amplitude and time shift between input and output are recorded. This measurement is repeated at different frequencies to obtain a complete spectrum of the frequency response of the tissue $[4,21]$.

The dynamic nanoindentation equipment applies an input oscillation in the form $h_{0}=h_{0, m}+$ $\Delta h_{0} \sin (2 \pi f t)$ to the free end of the cantilever (subscript 0 refers to the free end) and it measures the cantilever deflection $h_{c}=h_{c, m}+\Delta h_{c} \sin (2 \pi f t+\phi(2 \pi f))$, where $\phi(2 \pi f)$ is the time shift between input and output. In both the input and the output, the stationary term refers to the initial preload, while the time dependent part refers to the harmonic oscillation. Due to the additivity of the displacements, the penetration into the sample (subscript $s$ ) is obtained as $\Delta h_{s}=\Delta h_{0}-\Delta h_{c}$ [21]. It is important to note that the oscillation amplitude is much smaller than the initial preload displacement, i.e. $\Delta h_{s} \ll h_{s, m}$.

As for the viscoelastic materials, also for fluid filled tissues the storage and loss reduced moduli can be obtained from the dynamic mechanical analyses [2]:

$$
\begin{aligned}
& E^{\prime *}(f)=\frac{1}{2} \frac{\sqrt{\pi}}{\sqrt{A_{c}}}\left|\frac{\Delta F}{\Delta h_{s}}\right| \cos (\phi(2 \pi f)) \\
& E^{\prime \prime *}(f)=\frac{1}{2} \frac{\sqrt{\pi}}{\sqrt{A_{c}}}\left|\frac{\Delta F}{\Delta h_{s}}\right| \sin (\phi(2 \pi f))
\end{aligned}
$$

where $A_{c} \approx \pi R h_{s}$ is the contact area for shallow penetration depth $\left(h_{s} \ll R\right)$ and $\Delta F$ is the reaction force. The drained $(f \rightarrow 0)$ and the undrained $(f \rightarrow \infty)$ limits of the storage modulus are indicated as $E_{0}^{\prime *}$ and $E_{\infty}^{\prime *}$.

The dynamic nanoindentation experiments have been simulated in the frequency domain by modelling the coupled mechanical response of a fully saturated deformable anisotropic material [18]. Given the small harmonic oscillation of the indenter tip, a linearized anisotropic stress-strain rela-

tionship has been used. The fluid flow through the porous microstructure has been modelled using a Darcy type relationship.

\subsection{Dynamic nanoindentation of poroelastic solids: frequency domain analysis}

In this section the harmonic loading of a poroelastic medium in the frequency domain is briefly expounded.

In the finite element formulation of poroelasticity, the primary variables are the nodal displacements and the nodal fluid pressures. The set of governing equations are: the equilibrium equations, the kinematic compatibility, the mass conservation of fluid and the pressure - fluid velocity relationship; this latter is written in terms of the Darcy law:

$$
\mathbf{q}=-\mathbf{K} \nabla p
$$


where $\mathbf{q}$ is the fluid flux and $\mathbf{K}\left[\mathrm{m}^{4} /(N s)\right]$ is the permeability tensor.

The weak form of these equations can be more conveniently turned in a matrix equation as

$$
\left[\begin{array}{cc}
\mathbf{K}_{u u} & -\mathbf{G}_{u p} \\
\text { if } \mathbf{G}_{p u} & \mathbf{K}_{u u}
\end{array}\right]\left[\begin{array}{c}
\mathbf{U}_{n} \\
\mathbf{P}_{n}
\end{array}\right]=\left[\begin{array}{c}
\mathbf{F}_{u} \\
\mathbf{F}_{p}
\end{array}\right]
$$

that defines a sparse non symmetric system of linear equations. In equation (4) all terms are integrals over the domain on all the finite elements of the mesh as shown in Taffetani et al. [18]. This system is closed by the set of boundary conditions that are imposed on the axisymmetric domain as follows: i) the displacement component perpendicular to the axis of symmetry is zero; ii) the displacement component perpendicular to the base of the sample is zero; iii) the axis of symmetry, the bottom boundary and the indenter-sample contact region are impervious boundaries (i.e. the pressure gradient along the direction $\mathbf{n}$ normal to the boundary is zero: $\frac{d p}{d \mathbf{n}}=0$ ), while $p=0$ is imposed elsewhere; iv) the indenter is assumed to be infinitely stiff with respect to the sample, so that a displacement-based boundary condition on the region of the sample surface in contact with the indenter is used to simulate its action.

All the quantities shown in equation (1) are extracted from the simulations; in particular, the reaction force $\Delta F$ is obtained as the vertical resultant on the nodes that are in contact at the indenter-sample interface.

\subsection{Anisotropic mechanical model}

As the amplitude of the harmonic displacement of the indenter is small with respect to the pre-load displacement, small displacements and small strains theory is used. Let us consider a cylindrical reference system whose orthogonal directions are indicated with the subscripts $r, \theta$ and $a$, being, respectively, the radial, the hoop and the axial directions. The material is assumed to be transversely isotropic with its constitutive relationship defined as $\boldsymbol{\epsilon}=\mathbf{D} \boldsymbol{\sigma}$, where $\boldsymbol{\sigma}$ and $\boldsymbol{\epsilon}$ are, respectively, the stress and strain tensors written in Voigt notation $\left(\boldsymbol{\sigma}^{T}=\left[\sigma_{r r}, \sigma_{a a}, \sigma_{\theta \theta}, \sigma_{r a}\right]\right.$ and $\left.\boldsymbol{\epsilon}^{T}=\left[\epsilon_{r r}, \epsilon_{a a}, \epsilon_{\theta \theta}, \epsilon_{r a}\right]\right)$ and $\mathbf{D}$ is the compliance matrix, explicitly written as:

$$
\mathbf{D}=\frac{1}{E_{a}}\left[\begin{array}{cccc}
\lambda & -\nu & -\lambda \eta & 0 \\
-\nu & 1 & -\nu & 0 \\
-\lambda \eta & -\nu & \lambda & 0 \\
0 & 0 & 0 & \frac{E_{a}}{G}
\end{array}\right]
$$

The parameter $\lambda=E_{a} / E_{r}$ indicates the tissue anisotropy ratio as the ratio between the axial stiffness $E_{a}$ and the in-plane stiffness $E_{r}$; the parameters $\eta$ and $\nu$ represent, respectively, the outof-plane Poisson's ratio and the in-plane Poisson's ratio, while $G$ is the shear modulus.

The anisotropic permeability tensor $\mathbf{K}$ can be explicitly written as

$$
\mathbf{K}=\left[\begin{array}{lll}
K_{r r} & K_{r a} & K_{r \theta} \\
K_{a r} & K_{a \theta} & K_{a a} \\
K_{\theta r} & K_{\theta a} & K_{\theta \theta}
\end{array}\right]
$$

Since we assume axisymmetric properties, the axial direction identifies the principal material direction and the $(r, \theta)$ plane is the plane of isotropy, so that $K_{\theta \theta}=K_{r r}$.

It is useful to introduce two parameters related to the permeability: the anisotropy ratio $A_{k}=$ $K_{a a} / K_{r r}$ and the average permeability $\bar{K}=\left(K_{a a}+2 K_{r r}\right) / 3$. They allow to relate axial and radial permeabilities as

$$
K_{r r}=\frac{3 \bar{K}}{A_{k}+2} ; K_{a a}=A_{k} K_{r r}
$$




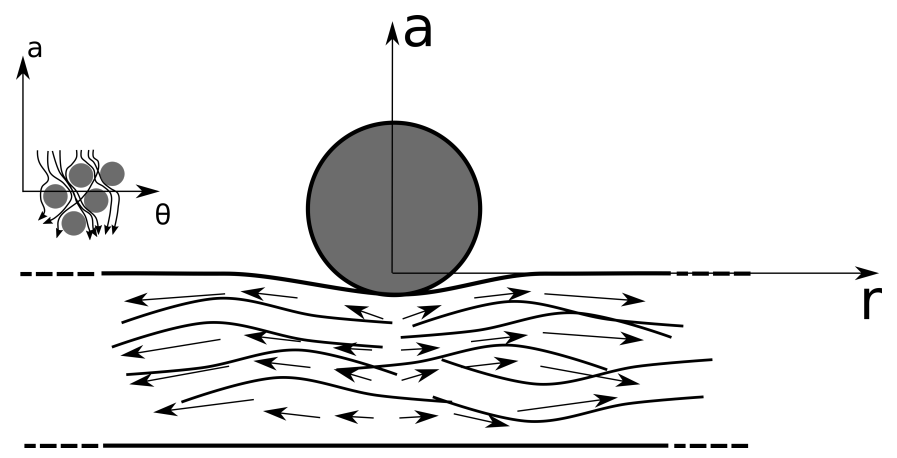

Figure 1: Schematic view of the anistropic fibers arrangement in a collagenouos tissue under a spherical indenter. Arrows indicate the preferred direction for fluid flow in the $(a, r)$ plane and (inset) in the $(a, \theta)$ plane.

As shown in Reynaud and Quinn [15] $A_{k}$ may vary through the tissue depth, from $A_{k}<1$ in the superficial layers to $A_{k}>1$ in the deep layers. In the superficial layers, this behaviour is due to the fiber arrangement that causes the fluid to flow more easily along the fiber direction, i.e. parallel to the articulating surface, than through the thickness; therefore $A_{k}<1$ is expected in our study (see figure 1). In order to determine a permeability tensor that explicitly takes into account the architecture of the fiber network, we consider a fiber orientation distribution of the form [20]:

$$
\rho(\theta)=\rho_{f} \frac{\bar{\rho}}{\rho_{0}}=\frac{\rho_{f}}{\rho_{0}}\left[\left(\frac{\sin (\theta)}{\tilde{A}}\right)^{2}+\left(\frac{\cos (\theta)}{\tilde{B}}\right)^{2}\right]^{-\beta}=\left[\left(\frac{\sin (\theta)}{A}\right)^{2}+\left(\frac{\cos (\theta)}{B}\right)^{2}\right]^{-\beta}
$$

where the parameters $\tilde{A}, \tilde{B}, \beta$ and $\rho_{0}$ are not independent but they have to fulfill the constraint $\int_{0}^{2 \pi} \int_{0}^{\pi} \frac{\rho \bar{\theta})}{\rho_{0}} \frac{1}{3} \sin (\theta) d \theta d \phi=1$. Parameters $A=3.7, B=0.07$ and $\beta=2.1$ for the superficial layers of bovine cartilage tissue have been found in a previous study [21] and they will be considered as given hereafter.

The global permeability tensor in equation (6) can be derived introducing a local anisotropic permeability tensor at the micro-scale as done in Federico and Herzog [3]. Let us consider a unit cell composed of a single straight collagen fiber surrounded by a cylindrical volume of matrix, such that the local (or microscopic) axial direction is the one along the fiber and the fiber density is denoted by $\phi$. For this unit cell, the transversely isotropic permeability becomes

$$
\mathbf{k}_{f}=\left[\begin{array}{ccc}
k_{\|} & 0 & 0 \\
0 & k_{\perp} & 0 \\
0 & 0 & k_{\perp}
\end{array}\right]=k_{0}(1-\phi)\left[\begin{array}{ccc}
1 & 0 & 0 \\
0 & 1-\phi & 0 \\
0 & 0 & 1-\phi
\end{array}\right]
$$

where $k_{\|}$and $k_{\perp}$ denote the permeabilities in the directions parallel and perpendicular to the fiber, respectively; $k_{0}$ is the isotropic permeability of the GAGs matrix and $\bar{\chi}_{f}=\frac{k_{\perp}}{k_{\|}}=1-\phi$ is the anisotropy ratio at the fibrillar level.

The macroscopic anisotropic permeability $\mathbf{K}$ is obtained by integrating the local tensor $\mathbf{k}_{f}$ on a unit sphere that spans all spatial directions weighted by the function $\rho(\theta)$ as:

$$
\mathbf{K}=\int_{0}^{2 \pi} \int_{0}^{\pi} \rho(\theta) \mathbf{R}^{T}(\theta) \mathbf{k}_{f} \mathbf{R}(\theta) \frac{1}{3} \sin (\theta) d \theta d \alpha=\frac{2 \pi}{3} \int_{0}^{\pi} \rho(\theta) \mathbf{R}^{T}(\theta) \mathbf{k}_{f} \mathbf{R}(\theta) \sin (\theta) d \theta .
$$

In this relation, axisymmetry has been used and the rotation tensor $\mathbf{R}=[\sin \theta, \cos \theta ;-\cos \theta, \sin \theta]$ rotates the local microscopic permeability tensor $\mathbf{k}_{f}$ onto the fiber global orientation $\theta$. The definition of the macroscopic permeability tensor in equation (10) is crucial in our analysis: it provides 


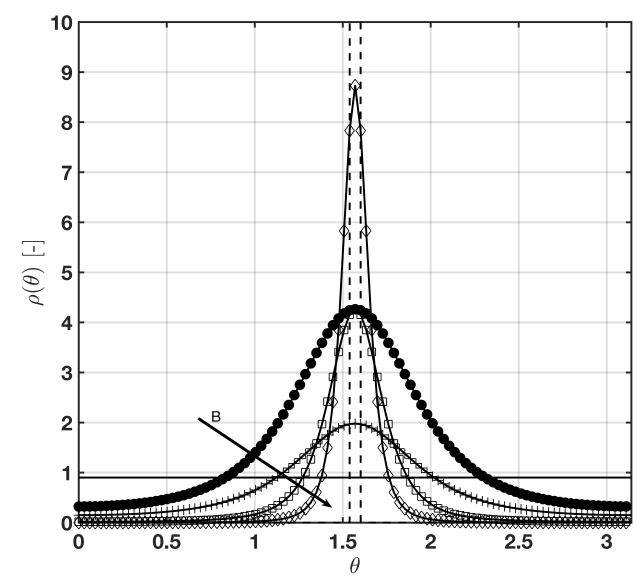

(a)

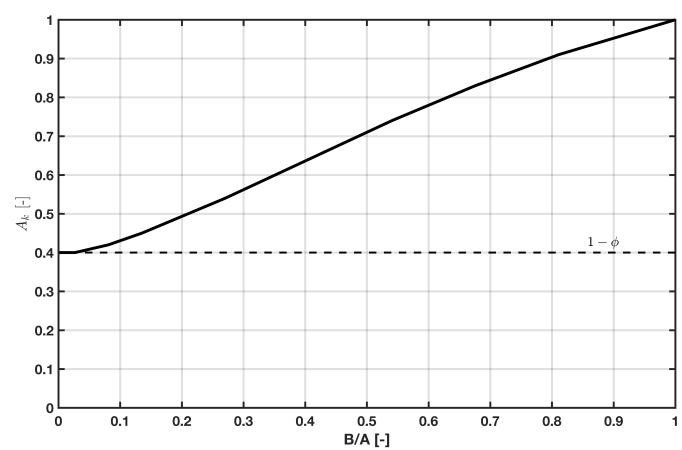

Figure 2: (a) Distribution of the collagen fiber orientation as a function of the angle $\theta, \theta=0$ being the axial direction; different curves refer to different values of the structural parameter $B$ in equation (8). (b)Dependence of the macroscopic anisotropy ratio $A_{k}$ on the anisotropy in the fibers distribution (solid line); the microscopic anisotropy ratio (dashed line) is consistenlty recovered when $B \rightarrow 0$, i.e. the fibers are parallel to the articular surface.

the possibility to infer the microscopic behaviour of the tissue (through $k_{0}$ and $\phi$ ), once the macroscopic identification of $\bar{K}$ and $A_{k}$ is given. In the following, experimental results will be fitted by identifying the parameters $A_{k}$ and $\bar{K}$.

Figure 2a shows the fiber orientation distribution for different values of the structural parameter $B$ : when $B=A$ the fibers are randomly oriented, while a decreasing value of $B$ results in a fiber distribution that narrows along the direction parallel to the articular surface $(\theta=\pi / 2)$. Figure $2 \mathrm{~b}$ shows the macroscopic anisotropy ratio of the permeability computed as $A_{k}=K_{a a} / K_{r r}$, i.e. the axial to transverse permeability ratio calculated at the macroscopic level through the integral (10) with $A=3.7$ and $\beta=2.1$. The anisotropy ratio is equal to the unity (i.e. isotropic permeability tensor) when $B=A$ and it decreases as $B$ decreases. When $B \rightarrow 0$ the fibers tend to be perfectly aligned tangentially to the articular surface and the permeability reaches its most anisotropic condition: macroscopically we obtain $A_{k}=\bar{\chi}_{f}=1-\phi$, which coincides with the fiber scale anisotropic ratio.

\section{Results}

In our previus analysis [18] we presented an extensive parametric study on the role of elastic anisotropy $\lambda$ in the dynamic response of articular cartilage, showing that the ratio $E_{0}^{\prime *} / E_{\infty}^{\prime *}$ decreases when the anisotropic elastic ratio $\lambda$ decreases. In other words, an anisotropic tissue (with axial elastic modulus lower than radial elastic modulus) exhibits a quasi-static to undrained storage modulus ratio which is lower than that of isotropic tissues. Furthermore, a more compliant axial direction also implies a higher frequency at the peak of the loss modulus if compared to isotropy. A decreasing value of the Poisson's ratio brings to a decreasing value of the peak of loss modulus $(\tan (\phi))$; for small value of $\lambda$, the effect of Poisson's ratio is less relevant.

In the present paper, instead, we focus on the anisotropy of the permeability: first we perform a parametric study on its effect on the mechanical properties of the articular cartilage; than, we carry out a parameters identification for three sets of experimental data. 
For the former we investigate a range of the anisotropy ratio of permeability varying in $A_{K}=$ $[0.1-10]$ while the tip radii considered are $R=[400 ; 125 ; 25 ; 7.5] \mu \mathrm{m}$. The initial preload displacement is set to $h_{0}=R / 10$ for all cases so we can make a comparison at the same representative indentation strain. A reference set of elastic parameters is assumed for this parametric analysis: $E_{a}=330 K P a ; \lambda=0.15 ; \nu=0.22$ and $\eta=\nu$.

For the latter, we consider the set of experimental results described in Taffetani et al. [21] where the superficial layer of bovine cartilage is tested with the dynamic nanoindentation using a $R=7.5 \mu \mathrm{m}$ spherical tip and three different pre-load penetration depths: $520 \mathrm{~nm}, 920 \mathrm{~nm}$ and $1200 \mathrm{~nm}$. The frequency ranges in the interval $[0-200 \mathrm{~Hz}]$.

As detailed in [18], the numerical simulations are conducted using a two steps approach: first the axisymmetric base state accommodated by the sample at the given mean indentation $h_{0, m}$ is computed using the displacement based finite element code Abaqus 6.8-EF1 (Simulia, Providence, RI, USA); then, the deformed geometry is used as input for the frequency domain model implemented into a Matlab environment (MathWorks, Natick, MA, USA).

\subsection{Parametric Study}

Figure 3a shows the typical frequency spectrum of the storage modulus in a dynamic nanoindentation simulation with a $7.5 \mu \mathrm{m}$ tip radius and different permeability anisotropy ratios (while average permeability is kept constant). Consistently with a previous study [18], we find that both the quasi static condition (the low frequency limit in the spectrum) and the undrained condition (the high frequency limit in the spectrum) do not depend on the permeability and in particular on its anisotropy ratio. In the frequency range from $10 \mathrm{~Hz}$ to $10^{4} \mathrm{~Hz}$, a progressive shift towards higher frequencies is observed for decreasing anisotropy ratio. In particular, the effect of $A_{k}$ is more substantial in the range $A_{k}>1$ than that observed in the range $A_{k}<1$. With a specific focus on the superficial layers of articular cartilage $\left(A_{k}<1\right)$, the sensitivity analysis reveals that a given storage modulus occurs at higher frequency than that for isotropic permeability.

Figure $3 \mathrm{~b}$ shows the typical spectrum for the time shift $(\tan (\phi))$ in a dynamic nanoindentation simulation with a $7.5 \mu \mathrm{m}$ tip radius and different permeability anisotropy ratios (while average permeability is kept constant). Here the variation of $A_{k}$ has an appreciable effect on two main factors: i) the maximum time shift (the peak of the curves) and ii) the frequency at this maximum of the time shift. In particular, both the peak value of the time shift and the frequency at peak decrease when the anisotropy ratio is decreased.

Figure 4a shows the frequency at the maximum time shift against the permeability anisotropy ratio for different tip radii: it decreases with decreasing $A_{k}$ and, consistently with the case of isotropic permeability, with increasing tip radius. Normalizing the frequency as $\bar{f}=f\left(\left(R h_{s}\right) /\left(E_{0}^{\prime *} \bar{K}\right)\right)[18]$, all the curves collapse as shown in figure $4 \mathrm{~b}$, ruling out the effect of the geometrical parameters. On the other side, the maximum of the tangent of the shift is not affected by the tip radius as shown in figure 5.

\subsection{Parameter identification}

The parameter identification is performed using the same approach followed in Taffetani et al. [21]. Figures $6 \mathrm{a}$ and $6 \mathrm{~b}$ show the agreement between the experimental data (markers) and the best fitting (lines) for the storage modulus (a) and $\tan (\phi)$ (b) versus the frequency of the applied load at the three different average penetration depths.

With respect to the different penetration depths we find: (i) the storage modulus at $f \rightarrow 0$ remains constant (within the experimental scatter) while $E^{* *}(200 H z)$ increases; (ii) the maximum dissipation (maximum of the curve $\tan (\phi)$ ) decreases with the penetration depth; (iii) the frequency 


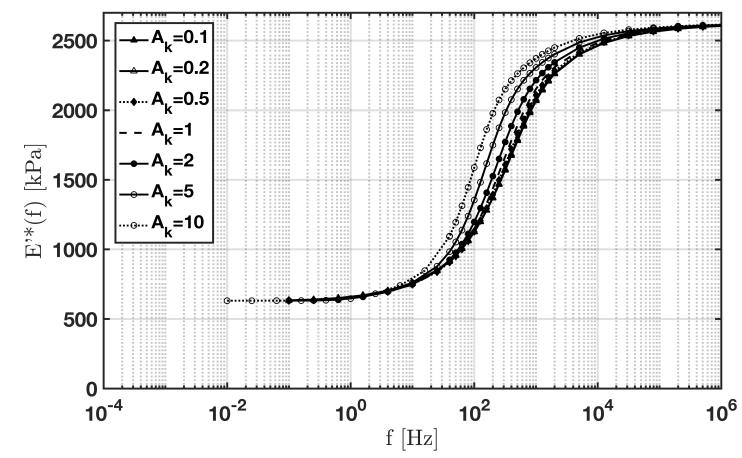

(a)

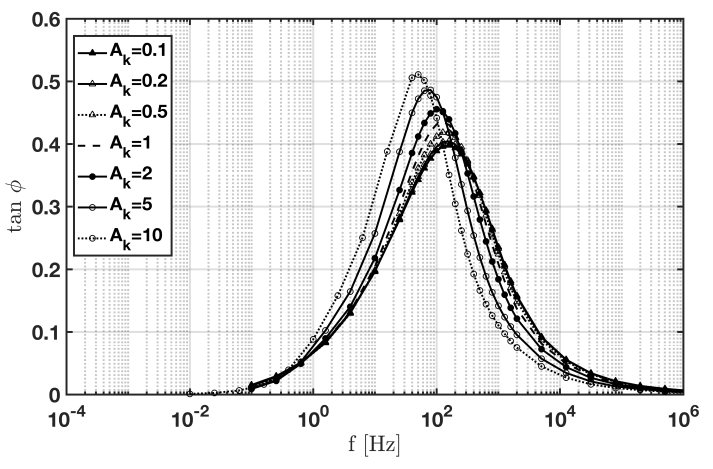

Figure 3: (a) Log-log plot of the storage modulus versus the loading frequency for a $R=7.5 \mu \mathrm{m}$ tip; each curve refers to a different anisotropic permeability ratio $A_{k}$. (b) Log-log plot of the tangent of the phase shift $\tan (\phi)$ versus the loading frequency for a $R=7.5 \mu \mathrm{m}$ tip; each curve refers to a different anisotropic permeability ratio $A_{k}$.
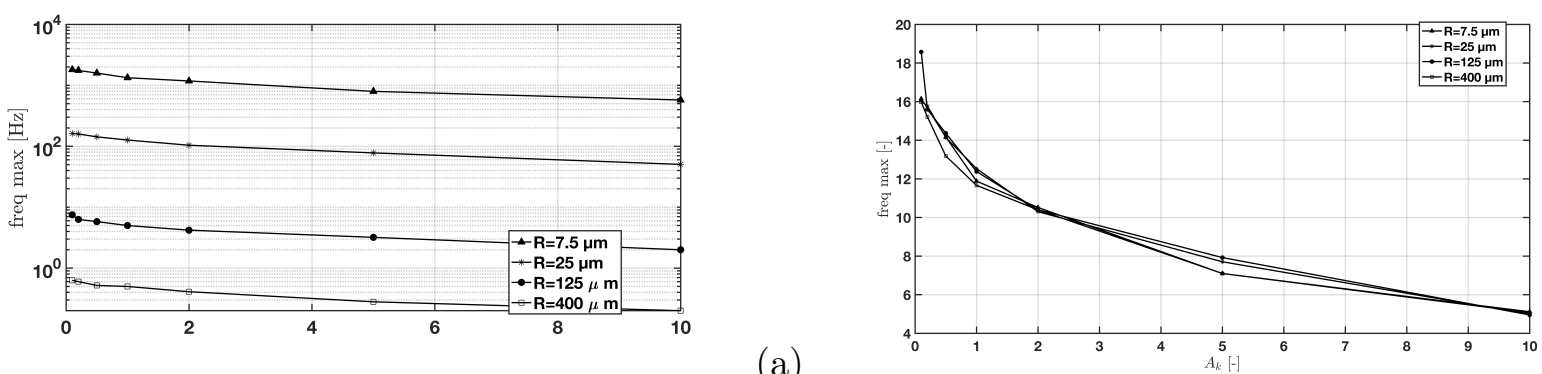

Figure 4: (a) Frequency at peak value of the $\tan (\phi)$ signal as a function of $A_{k}$ for different tip radii. (b) Normalized frequency $\bar{f}=f \frac{R h_{s}}{E_{0}^{\prime \prime} \bar{K}}$ at the peak of the $\tan (\phi)$ signal as a function of $A_{k}$ for different tip radii. The four curves now collapse onto each other.

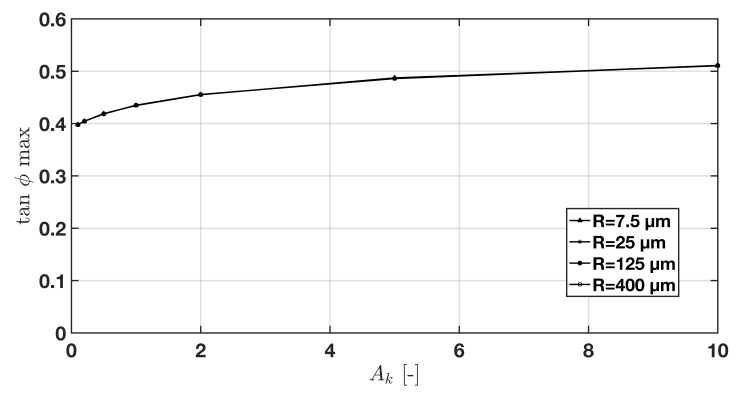

Figure 5: Maximum value of $\tan (\phi)$ as a function of $A_{k}$ for different tip radii collapses onto a single curve, thus revealing that it is not affected by the indenter radius $R$

at peak decreases with the penetration depth. The values of the fitted model parameters are reported in the table 1.

\section{Discussion}

In this work we assessed the effect of the anisotropic permeability on the mechanical response of the superficial layer of bovine cartilage, when subjected to dynamic AFM-based nanoindentation 


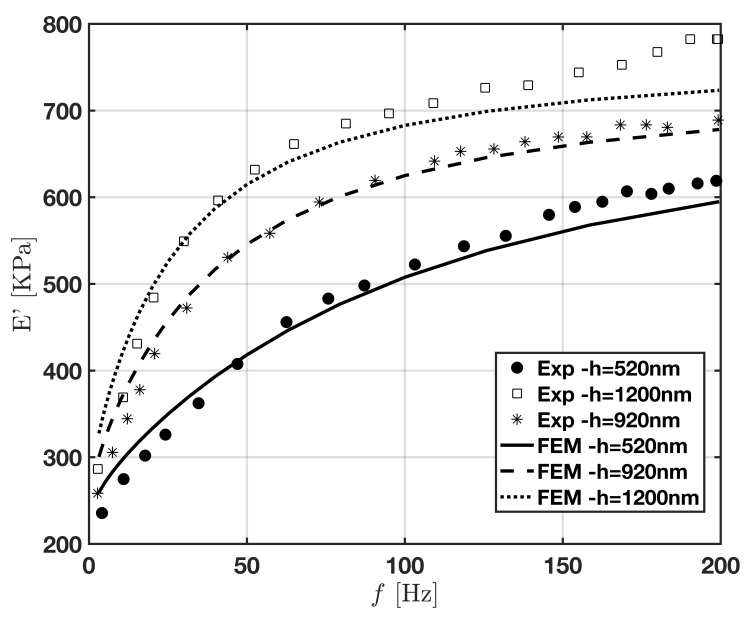

(a)

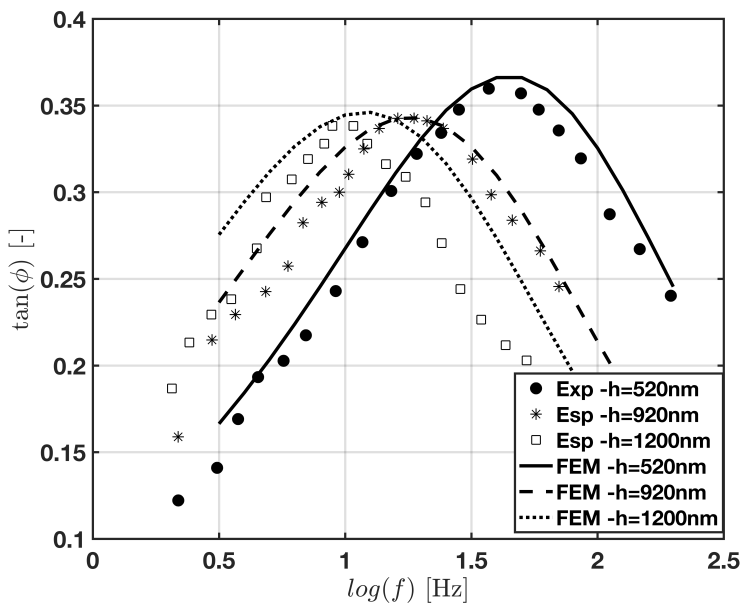

Figure 6: (a) Storage modulus and (b) tangent of loss angle $\phi$ against the loading frequency; markers refer to the experimental data, solid curves refer to the finite element model fittings. Results are reported for the three penetration depths, $h_{s}=520 \mathrm{~nm}, h_{s}=920 \mathrm{~nm}$ and $h_{s}=1200 \mathrm{~nm}$, obtained with the same $R=7.5 \mu \mathrm{m}$ spherical indenter.

Table 1: Parameters of the numerical model identified for the three penetration depths.

\begin{tabular}{|c|c|c|c|c|c|c|}
\hline$h_{s}[\mathrm{~nm}]$ & $E[K P a]$ & $G[K P a]$ & $\nu$ & $\lambda$ & $\bar{K}\left[x 10^{-15} \mathrm{~m}^{4} /(\mathrm{Ns})\right]$ & $A_{k}$ \\
\hline \hline 520 & 162 & 81 & 0.065 & 0.1 & 2 & 0.4 \\
920 & 165 & 82.5 & 0.09 & 0.1 & 1.5 & 0.3 \\
1200 & 170 & 85.5 & 0.075 & 0.1 & 1.2 & 0.25 \\
\hline
\end{tabular}

tests.

The AFM-based dynamic nanoindentation has confirmed to be an effective experimental test to assess mechanical (elastic constants) as well as physical parameters (such as permeability) including their anisotropic features. Although anisotropic permeability has been already assessed macroscopically through more classical compression tests, see for example [15], here we analysed its properties at a smaller length scale confining ourself to the superficial layer of AC by means of shallow AFMbased nanoindentation. In a previous work [21] we proved the effectiveness of this experimental technique in evaluating the anisotropy in elastic properties, while here we showed its capability to investigate the anisotropy of the tissue hydraulic permeability. Although the nanoindentation tests have been performed along one single direction (perpendicular to the articular surface), the possibility to tune the characteristic sizes and the characteristic times by selecting the penetration depth and the frequency of load, allows one to obtain information also along the direction perpendicular to the direction of load.

Our sensitivity analysis showed that the permeability anisotropy ratio affects the peak value of the phase shift. Its role can be singled out by introducing the normalized frequency $\bar{f}=$ $f\left(\left(R h_{s}\right) /\left(\bar{K} E_{0}^{\prime *}\right)\right)$ that allows one to rule out the effect of the characteristic size. On the contrary, the drained (low frequency limit) and the undrained (high frequency limit) extrema of the storage modulus do not depend on the permeability and on its anisotropy ratio, since they are affected only by the anisotropic elastic properties. Crucially, this preliminary analysis allows us to carry out the subsequent identification process with few iterations of a trial and error procedure. Before discussing it, we want to point out that our analysis considers a linearized anisotropic material model, not allowing for the finite strain state that may occur in the tissue and that would induce local axis 
rotation of the material elastic stiffness and permeability tensors. This limitation has been partly treated previously [21] where local rotation of material anisotropic elastic tensor is included: in this previous work we showed that this simplification does not affect the overall capability of the model to simulate the experimental response, in particular when shallow penetration depths are considered as in the cases studied in this paper, where the maximum penetration depth is $16 \%$ of the tip radius.

With the identification analysis we estimated the anisotropic mechanical and physical properties of the tissue by fitting the experimental data in terms of the storage modulus and phase shift signals in a frequency range of $[0-200 \mathrm{~Hz}]$, using a finite element model that solves the AFM nanoindentation problem in the frequency domain. The frequency range analysed in this work is of practical relevance both for regular gait conditions and for high strain rate loading, as those occuring during injuries or sport activities [1].

In the model considered in this paper, the poroelastic approach presented in Taffetani et al. [18] is enriched with the implementation of an anisotropic permeability tensor that accounts for the fiber induced anisotropic fluid flow. We assume that the fiber distribution is equal to the one found in [21] for the superficial layers of cartilage and we rely on the discussion therein provided when we assume that this approach is well suited to simulate the AFM dynamic nanoindentation (although the fibrous structure is not explicitly modelled).

The elastic constants $E_{a}$ and $G$ are found slightly increasing with penetration depth, consistently with the typical mechanical response of the tissue that features increasing stiffness with increasing applied strain. In this augmented model, however, a value of anisotropic ratio $\lambda=0.1$ less than the value found with our previous model (0.16 [21]) was identified. Although the difference appears small, the value is now closer to that found in previous investigations [3], thus supporting the initial hypothesis that the introduction of an anisotropic permeability is required toward a finer characterization of the tissue.

In our procedure we identify the macroscopic quantities related to the permeability, i.e. the mean value $\bar{K}$ and the anisotropy ratio $A_{k}$. Both of them are found to decrease with the penetration depth of the spherical indenter: this finding is consistent with a compaction of the fibers upon compression along the axial direction, that would entail an increase in the fiber volume fraction along the thickness direction while decreasing it in the transverse direction. With respect to the model used in Taffetani et al. [21], here we introduce an additional degree of freedom related to the (macroscopic) permeability while fixing the relation between macroscopic and microscopic properties through the equations (10). The trends found for $\bar{K}$ and $A_{k}$ are consistent with (i) a constant microscopic $k_{0}\left(h_{s}\right)=$ const $=4.3 \cdot 10^{-16} \mathrm{~m}^{4} /(\mathrm{Ns})$ and (ii) a depth dependent fiber compaction as $\phi\left(h_{s}=520\right)=0.6, \phi\left(h_{s}=920\right)=0.7$ and $\phi\left(h_{s}=1200\right)=0.75$. Recalling that the applied strain $\epsilon_{c}$ can be estimated as $\epsilon_{c} \approx 0.2 r\left(h_{s}\right) / R[19]$ with $r\left(h_{s}\right)=\sqrt{2 R h_{s}-h_{s}^{2}}$ being the contact radius, this enriched model allows us to propose a discrete relation between the microscopic fiber compaction and applied strain. The simplifying assumption that the intrinsic compressibility of the collagen fiber itself is negligible with respect to the compressibility of the GAGs matrix would support the linear relationship between the fiber volume fraction and the applied strain as shown in figure 7 , along with the linear relationship between the anisotropic ratio $A_{k}$ and $\phi$.

Due to the complex microstructure of $\mathrm{AC}$, its response depends on the testing conditions and the length scales investigated. Hence, a careful comparison of the present analysis with results available in literature is required, with a particular focus on the testing conditions. Reynaud et al. $[15,16]$ developed a technique for the direct measurement of hydraulic permeability in statically compressed disks of adult bovine cartilage. They found that the hydraulic permeability remains nearly isotropic for static compressions less than 10\%. Above this threshold, the permeability decreases, more in the radial component than in the axial one (a 10-fold difference between axial and 


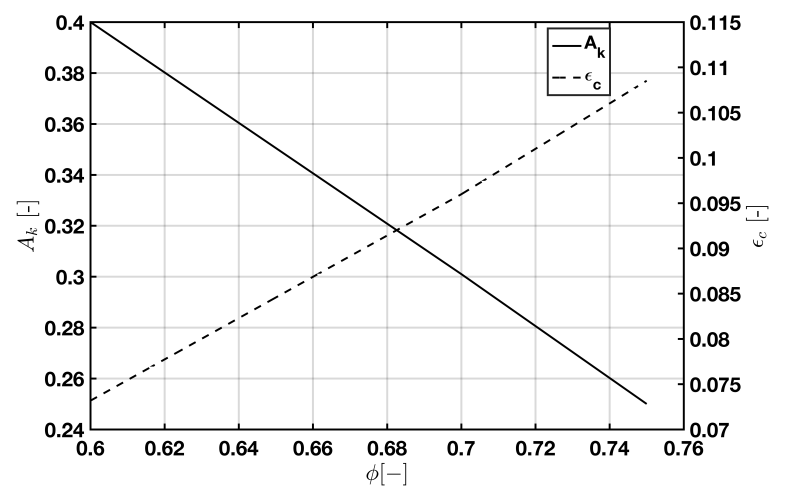

Figure 7: (left axis) Macroscopic permeability ratio $A_{K}$ obtained using the parameters from the best fitting for the three penetration depths investigated against the volumetric fraction of collagen fibers, this latter estimated using the integral 10. (right axis) Indentation strain, computed as $\epsilon_{c} \approx 0.2 \sqrt{2 R h_{s}-h_{s}^{2}} / R[19]$ versus the volumetric fraction of collagen fibers.

radial directions): a strong anisotropy in the hydraulic permeability tensor is then evident for static compression of 20-40\%. In the present model we predict that the anisotropy ratio $A_{k}=K_{a a} / K_{r r}$ is decreasing with increasing penetration depth; but, for the three penetration depths considered in this study, the axial permeability decreas was $60 \%$, while the transverse component decrease was only $36 \%$ when moving both from $520 \mathrm{~nm}$ to $920 \mathrm{~nm}$ and from $920 \mathrm{~nm}$ to $1200 \mathrm{~nm}$ depth (see figure 8). This discrepancy with respect to the Reynaud's study can be ascribed to the different sizes investigated in these two studies. In the present work only the superficial layer of AC was investigated by means of shallow penetration depth of the indenter (the largest penetration depth is $1200 \mathrm{~nm}$ ), which is small enough to probe a space encompassing few collagen fibers along the thickness direction. So, only the superficial permeability is measured. In Reynaud and Quinn [15] the superficial layer (top $100 \mu \mathrm{m}$ ) was removed and a thick $(900 \mu \mathrm{m})$ sample was considered, thus including the middle zone where an isotropic permeability is expected. Therefore, they measured a global permeability.

Federico and Herzog [3] studied the anisotropic permeability at two length scales: (i) the scale of the single collagen fiber surrounded by the GAG matrix and (ii) the scale of the fiber network. In this latter regime, they found an anisotropic ratio for the superficial layers of about 0.58 when a volume fraction of the fiber of $60 \%$ is given. This value is higher than that found in this work $(\bar{\chi}=0.4)$ and the discrepancy is due to the fiber orientation distribution assumed in Federico and Herzog study, which includes a higher volumetric fraction of fibers oriented along direction close to $\theta=0$ (perpendicular to the articular surface direction); in this work a much narrower orientation distribution around the direction $\theta=\pi / 2$ has been considered. At this larger scale, the permeability anisotropy ratio is strictly related to the fiber orientation distribution. 


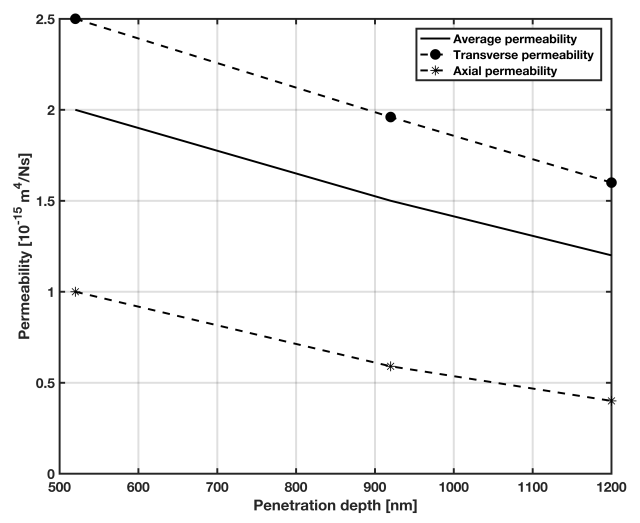

Figure 8: Axial permeability $K_{a a}$ and transverse permeability $K_{r r}$ obtained using the parameters from the best fitting for the three penetration depths investigated. Average permeability $\bar{K}=$ is also reported for comparison.

\section{Acknowledgements}

The Authors wish to thank the student Andrea Chiara De Nadai for performing part of the numerical analyses presented in this paper.

\section{References}

[1] J. Buckwalter. Articular cartilage injuries. Clinical orthopaedics and related research, 402:21$37,2002$.

[2] Y.-T. Cheng, W. Ni, and C.-M. Cheng. Nonlinear analysis of oscillatory indentation in elastic and viscoelastic solids. Physical review letters, 97(7):075506, 2006.

[3] S. Federico and W. Herzog. On the anisotropy and inhomogeneity of permeability in articular cartilage. Biomechanics and modeling in mechanobiology, 7(5):367-378, 2008.

[4] O. Franke, K. Durst, V. Maier, M. Göken, T. Birkholz, H. Schneider, F. Hennig, and K. Gelse. Mechanical properties of hyaline and repair cartilage studied by nanoindentation. Acta Biomaterialia, 3(6):873-881, 2007.

[5] O. Franke, M. Göken, M. Meyers, K. Durst, and A. Hodge. Dynamic nanoindentation of articular porcine cartilage. Materials Science and Engineering: C, 31(4):789-795, 2011.

[6] L. Han, E. H. Frank, J. J. Greene, H.-Y. Lee, H.-H. K. Hung, A. J. Grodzinsky, and C. Ortiz. Time-dependent nanomechanics of cartilage. Biophysical journal, 100:1846-1854, 2011.

[7] K. Hu, P. Radhakrishnan, R. Patel, and J. Mao. Regional structural and viscoelastic properties of fibrocartilage upon dynamic nanoindentation of the articular condyle. Journal of Structural Biology, 136(1):46-52, 2001.

[8] Y. Hu, E. Chan, J. Vlassak, and Z. Suo. Poroelastic relaxation indentation of thin layers of gels. Journal of Applied Physics, 110(8):086103-1:3, 2011.

[9] Y. Hu, X. Zhao, J. J. Vlassak, and Z. Suo. Using indentation to characterize the poroelasticity of gels. Applied Physics Letters, 96(12):121904, 2010. 
[10] C. Y. Huang, V. C. Mow, and G. A. Ateshian. The role of flow-independent viscoelasticity in the biphasic tensile and compressive responses of articular cartilage. Journal of biomechanical engineering, 123:410-417, 2001.

[11] H. J. Mankin, V. C. Mow, J. A. Buckwalter, J. P. Iannotti, and A. Ratcliffe. Articular cartilage structure, composition, and function. Orthopaedic basic science: biology and biomechanics of the musculoskeletal system, 2:443-70, 2000.

[12] A. Maroudas. Physicochemical properties of cartilage in the light of ion exchange theory. Biophysical journal, 8:575-595, 1968.

[13] A. D. Pearle, R. F. Warren, and S. A. Rodeo. Basic science of articular cartilage and osteoarthritis. Clinics in sports medicine, 24:1-12, 2005.

[14] T. Quinn, P. Dierickx, and A. Grodzinsky. Glycosaminoglycan network geometry may contribute to anisotropic hydraulic permeability in cartilage under compression. Journal of biomechanics, 34(11):1483-1490, 2001.

[15] B. Reynaud and T. M. Quinn. Anisotropic hydraulic permeability in compressed articular cartilage. Journal of biomechanics, 39(1):131-137, 2006.

[16] B. Reynaud and T. M. Quinn. Tensorial electrokinetics in articular cartilage. Biophysical journal, 91:2349-2355, 2006.

[17] M. E. Stender, R. A. Regueiro, and V. L. Ferguson. A poroelastic finite element model of the bone-cartilage unit to determine the effects of changes in permeability with osteoarthritis. Computer methods in biomechanics and biomedical engineering, 20(3):319-331, 2017.

[18] M. Taffetani, E. Bertarelli, R. Gottardi, R. Raiteri, and P. Vena. Modelling of the frequency response to dynamic nanoindentation of soft hydrated anisotropic materials: Application to articular cartilage. CMES - Computer Modeling in Engineering and Sciences, 87(5):433-460, 2012.

[19] M. Taffetani, R. Gottardi, D. Gastaldi, R. Raiteri, and P. Vena. Poroelastic response of articular cartilage by nanoindentation creep tests at different characteristic lengths. Medical Engineering and Physics, 36(7):850-858, 2014.

[20] M. Taffetani, M. Griebel, D. Gastaldi, S. Klisch, and P. Vena. Poroviscoelastic finite element model including continuous fiber distribution for the simulation of nanoindentation tests on articular cartilage. Journal of the Mechanical Behavior of Biomedical Materials, 32:17-30, 2014.

[21] M. Taffetani, R. Raiteri, R. Gottardi, D. Gastaldi, and P. Vena. A quantitative interpretation of the response of articular cartilage to atomic force microscopy-based dynamic nanoindentation tests. Journal of Biomechanical Engineering, 137(7):071005:1-8, 2015. 